


\begin{tabular}{|c|c|c|c|}
\hline$\Omega$ & 廿 & $\equiv$ & (2) \\
\hline Giovanni Sotgiu, & For affiliations, see & Stefano Aliberti: & stefano.aliberti@unimib.it \\
\hline Stefano Aliberti, & Acknowledgments section & $\begin{array}{l}\text { Dipartimento di Scienze } \\
\text { della Salute, Università degli }\end{array}$ & \\
\hline Delia Goletti, & & Studi di Milano-Bicocca, UO & \\
\hline Giovanni Battista & & Clinica Pneumologica, AO & \\
\hline Migliori & & 33, 20900, Monza, Italy & \\
\hline
\end{tabular}

\section{TB elimination: a dream or a reality? Key lessons from the ERS external course in Dubrovnik, Croatia}

Tuberculosis (TB) is one of the most important causes of death among infectious diseases, alongside with HIV/AIDS and malaria, despite the existence of a cost-effective strategy for its clinical management, prevention and control. TB elimination represents a crucial public health goal, which can be obtained by reducing the prevalence of individuals with latent TB infection, through a preventive therapeutic approach. By reducing the large pool of infected individuals, global TB incidence will be decreased and the target of TB elimination will be achieved by the year 2050, as advocated by the Millennium Development Goals and the Stop TB Partnership.

In mid-2013, the European Respiratory Society (ERS), in association with the FP7 EUfunded TB-PAN-NET project, ran an unique course on TB elimination, entitled "TB elimination: a dream or reality?", in Dubrovnik, Croatia. The course was attended by 35 participants who had the chance to discuss crucial topics on TB elimination with a faculty of 10 people from the ERS, the World Health Organization (WHO) and the American Thoracic Society.
During the discussion among participants and faculty, five points of interest were particularly developed.

\section{Epidemiological features helpful for eradication strategies}

The faculty and participants described the current epidemiological scenario. Estimates of the burden of disease, as well as that of latent TB infection (LTBI), were shown. The pros and cons of the current WHO strategy were discussed, focusing on potential solutions in the different WHO regions. The current rate of decline in global TB disease incidence, based on Bacille Calmette Guerin (BCG) vaccination as well as treatment of TB disease and of LTBI, will not allow the achievement of global elimination by 2050 . It will be important to scale up the WHO's public health strategies, as well as to improve surveillance activities worldwide.

- Suggested article: Dye C, Glaziou P, Floyd K, et al. Prospects for tuberculosis elimination. Annu Rev Public Health 2013; 34: 271-286.
Statement of Interest S. Aliberti has received payment for lectures from Zambon, Boehringer Ingelheim, Pfizer, Novartis, BRAHMS, GlaxoSmithKline, Menarini, Merck Sharp \& Dohme, Nycomed and Abbott.
HERMES syllabus link module: B. 4 


\section{New drugs for LTBI and TB disease}

Several presentations explained the main principles of the design of the experimental studies currently used to evaluate new drugs. The methodology used by pharmaceutical companies was evaluated, using practical examples. The participants were actively involved and prepared protocols to submit to the regulatory agencies to test their new skills.

In particular, the role of new study designs (i.e., adaptive trials) which can enable the evaluation of the safety and efficacy profile of several drugs, was discussed. During the discussion, it was pointed out that clinical and epidemiological analysis of individuals with comorbidities (mainly those with TB/HIV co-infection), and of those in early and advanced phases of their life, is crucial in evaluating drugs' utility.

- Suggested article: Wallis RS. Sustainable tuberculosis drug development. Clin Infect Dis 2013; 56: 106-113.

\section{New diagnostics for LTBI and TB disease}

The same approach adopted for the drug section was used to discuss diagnostics, pointing out current resources and new research paths towards innovative tools.

It is necessary to use an evidence-based approach to identify the best diagnostic method; unfortunately, there are several economic, political and technical issues that hinder the research and development into diagnostic innovations. One of the most relevant difficulties in the development of diagnostic methods for LTBI and TB disease is the lack of a validated surrogate marker; new genetic methods seem promising.

- Suggested article: Wallis RS, Kim P, Cole S, et al. Tuberculosis biomarkers discovery: developments, needs and challenges. Lancet Infect Dis 2013; 13: 362-372.

\section{Clinical management of LTBI and TB disease}

The faculty prepared electronic materials to describe the shortcomings of the treatment regimens currently prescribed for TB disease and LTBI. Numerous examples drawn from current practice were used to describe the correct clinical approach in the management of Mycobacterium tuberculosis infection. The recent evidence-based WHO guidelines represent an important clinical tool to manage difficult cases; it is crucial to prescribe a therapeutic combination tailored using drug susceptibility tests (DST) in order to avoid the emergence of further resistance.

- Suggested article: Falzon D, Jaramillo E, Schünemann $\mathrm{HJ}$, et al. WHO guidelines for the programmatic management of drugresistant tuberculosis: 2011 update. Eur Respir J 2011; 38: 516-528.

\section{Research priorities}

The last day of the course was dedicated to future possibilities for medical and nonmedical specialties in global TB elimination. TB represents a major, immediate public health problem that should be treated strategically with the involvement of stakeholders from a range of fields, taking in specialists in political affairs, public health, pulmonary medicine, infectious diseases, social medicine, statistics, etc., as well as partners such as the pharmaceutical industry.

New training and educational needs are likely to be identified in the near future and could be filled in by the ERS. New initiatives should be focused on the clinical management of difficult-to-treat cases as well as on public health activities to be implemented at national and sub-national levels.

\section{Acknowledgements}

Author affiliations are as follows. Giovanni Sotgiu: Epidemiology and Medical Statistics Unit, Department of Biomedical Sciences, University of Sassari - Research, Medical Education and Professional Development Unit, AOU Sassari; Stefano Aliberti: Department of Health Science, University of 
Milan Bicocca, AO San Gerardo, Monza; Delia Goletti: Translational Research Unit, Department of Epidemiology and Preclinical Research, "L. Spallanzani" National Institute for Infectious Diseases (INMI), IRCCS, Rome;
Giovanni Battista Migliori: World Health Organization Collaborating Centre for Tuberculosis and Lung Diseases, Fondazione S. Maugeri, Care and Research Institute, Tradate, Italy.

\section{Further reading}

1. Blasi F, Reichman LB, Migliori GB. Presenting the European Forum for TB Innovation: innovative thinking in progressing towards TB elimination in Europe. Eur Respir J 2012; 40: 806-808.

2. Diel R, Loddenkemper R, Zellweger JP, et al. Old ideas to innovate TB control: preventive treatment to achieve elimination. Eur Respir J 2013; 42: 785-801.

3. Dye C, Glaziou P, Floyd K, et al. Prospects for tuberculosis elimination. Annu Rev Public Health 2013; 34: $271-286$.

4. Falzon D, Jaramillo E, Schünemann HJ, et al. WHO guidelines for the programmatic management of drug-resistant tuberculosis: 2011 update. Eur Respir J 2011; 38: $516-528$.
5. Glaziou P, Falzon D, Floyd K, et al. Global epidemiology of tuberculosis. Semin Respir Crit Care Med 2013; 34: 3-16.

6. Schluger NW. Advances in the diagnosis of latent tuberculosis infection. Semin Respir Crit Care Med 2013; 34: 60-66.

7. Vernon A. Treatment of latent tuberculosis infection. Semin Respir Crit Care Med 2013; 34: 67-86.

8. Wallis RS, Kim P, Cole S, et al. Tuberculosis biomarkers discovery: developments, needs, and challenges. Lancet Infect Dis 2013; 13: 362-372.

9. Wallis RS. Sustainable tuberculosis drug development. Clin Infect Dis 2013; 56: 106-113. 